\title{
TRABAJO INTERCENTROS PARA LA DEFINICIÓN DE LA NUEVA ESTRUCTURA CURRICULAR DEL PLAN DE ESTUDIOS DE LA LICENCIATURA EN BIOLOGÍA: UNA VISIÓN POR COMPETENCIAS
}

\author{
Intercenter work for the definition of the new curricular structure of the study plan of \\ biology: a vision by competences
}

\begin{abstract}
Georgina Adriana Quiroz Rocha
Departamento de Botánica y Zoología. Centro Universitario de Ciencias Biológicas y Agropecuarias, Universidad de Guadalajara. Camino Ramón Padilla Sánchez 2100 Nextipac, 45200 Zapopan, Jal. *Autor para correspondencia: georgina.quiroz@academicos.udg.mx Liza Danielle Kelly Gutiérrez Departamento de Ciencias Biológicas. Centro Universitario de la Costa, Universidad de Guadalajara. Av. Universidad \#203, delegación Ixtapa, CP. 48280, Puerto Vallarta, Jalisco, México.

Rosio Teresita Amparán Salido

Departamento de Ciencias Biológicas. Centro Universitario de la Costa, Universidad de Guadalajara. Av. Universidad \#203, delegación Ixtapa, CP. 48280, Puerto Vallarta, Jalisco, México.

Rosa María Domínguez Arias

Departamento de Biología Celular y Molecular. Centro Universitario de Ciencias Biológicas y Agropecuarias, Universidad de Guadalajara. Camino Ramón Padilla Sánchez 2100 Nextipac, 45200 Zapopan, Jal.

Cinthya Araceli López López

Departamento de Ciencias Ambientales. Centro Universitario de Ciencias Biológicas y Agropecuarias, Universidad de Guadalajara. Camino Ramón Padilla Sánchez 2100 Nextipac, 45200 Zapopan, Jal.

Pedro Medina Rosas

Departamento de Ciencias Biológicas. Centro Universitario de la Costa, Universidad de Guadalajara. Av. Universidad \#203, delegación Ixtapa, CP. 48280, Puerto Vallarta, Jalisco, México. Alejandro Muñoz Urias

Departamento de Ecología. Centro Universitario de Ciencias Biológicas y Agropecuarias, Universidad de Guadalajara. Camino Ramón Padilla Sánchez 2100 Nextipac, 45200 Zapopan, Jal. Graciela Gudiño Cabrera

Departamento de Biología Celular y Molecular. Centro Universitario de Ciencias Biológicas y Agropecuarias, Universidad de Guadalajara. Camino Ramón Padilla Sánchez 2100 Nextipac, 45200

Zapopan, Jal.
\end{abstract}

\section{Resumen}

El presente documento ofrece un acercamiento al trabajo colegiado llevado a cabo para la reestructuración curricular del Programa Educativo de Pregrado de Licenciatura en Biología de la Universidad de Guadalajara. Presenta los principales elementos de análisis y definición curricular que dieron estructura a la propuesta de dictamen que actualmente se encuentra vigente. Finalmente, arroja algunas reflexiones en torno a los retos que implica el trabajo colegiado entre comités curriculares denominados "intercentros".

Palabras clave: Diseño curricular, programa educativo

\begin{abstract}
This document offers an approach to the collegiate work carried out for the curricular restructuring of the Undergraduate Educational Program for the Bachelor of Biology at the University of Guadalajara. It presents the main elements of analysis and curricular definition that gave structure to the opinion proposal that is currently in force. Finally, it provides some reflections on the challenges involved in collegiate work between curricular committees called "inter-centers".
\end{abstract}

Key words: Curriculum design, educational program 


\section{Introducción}

La estructura de la Universidad de Guadalajara se basa en un modelo de red conformado por seis centros universitarios temáticos, cuya sede se encuentra en la Zona Metropolitana de Guadalajara, y que son denominados así por estar integrados a partir de la especialización en un campo disciplinar; nueve centros universitarios regionales, situados en las distintas regiones del estado de Jalisco y con programas educativos interdisciplinarios; y un Sistema de Universidad Virtual con modalidad a distancia.

Los programas educativos que ofrece la Universidad de Guadalajara pueden ser ofertados en uno o varios de los centros universitarios, de tal manera que en el caso particular de la Licenciatura en Biología actualmente es ofertada en el Centro Universitario de Ciencias Biológicas y Agropecuarias (CUCBA), categorizado como centro temático, y en el Centro Universitario de la Costa (CUCosta), categorizado como centro regional ubicado en la ciudad de Puerto Vallarta Jalisco.

Ante esto, el proceso de reestructuración curricular debía desarrollarse con la participación de académicos y especialistas provenientes de ambos centros universitarios.

\section{Antecedentes de la Licenciatura en Biología}

La carrera de Biología de la Universidad de Guadalajara, se crea en la Facultad de Ciencias que a su vez se funda con dictamen del Consejo General Universitario en junio de 1980, con la iniciativa del Ing. Edmundo Ponce Adame, primer director de la Facultad. En 1990 se separa el Programa de la Licenciatura en Biología, de Física y Matemáticas y se instituye la Facultad de Ciencias Biológicas. En 1991 se establece el Centro de Estudios de la Costa como una dependencia descentralizada de la Facultad, con sede en San Patricio-Melaque, municipio de Cihuatlán, Jalisco (Universidad de Guadalajara, 2004).

En 1992, se proponen modificaciones al Plan de Estudios, con base en los nuevos paradigmas en el área de la Biología, siendo el Director de la Facultad de Ciencias Biológicas el Dr. Juan Luis Cifuentes Lemus. Se hace énfasis en el área de investigación, dicho plan de estudios originó la apertura de las orientaciones en Biología pesquera y Acuicultura en el Centro de Estudios de la Costa, Melaque; en Manejo de Recursos Naturales en el entonces Laboratorio Natural Las Joyas en la Sierra de Manantlán; la orientación en Sistemas limnéticos en el Instituto de Limnología en Chapala; gestión ambiental con sede en el Bosque Escuela La Primavera de la Facultad de Agronomía, y las orientaciones de Biodiversidad, Biología experimental y Enseñanza de la Biología con sede en la Facultad de Ciencias Biológicas. En 1995 inició la orientación en Biología Marina en Puerto Vallarta, con la creación del Centro Universitario de la Costa.

Con la consolidación de la Reforma Universitaria en diciembre de 1993, se establece la Red Universitaria, se adopta el modelo departamental y en mayo de 1994 se aprueba la creación del Centro Universitario de Ciencias Biológicas y Agropecuarias que en sus inicios se constituyó con las carreras de Ingeniero Agrónomo, Médico Veterinario y Zootecnista y Biología. La Facultad de Ciencias Biológicas se transformó en la División de Ciencias Biológicas y Ambientales. En 1995 se crean el Centro Universitario de la Costa y el Centro Universitario de la Costa Sur (Universidad de Guadalajara 2010) que siguen ofreciendo para Biología las orientaciones terminales. El 15 de junio de 1995 se propone al Consejo General Universitario la reestructuración del Plan de Estudios de la Licenciatura en Biología, se establece la tutoría académica, un semestre de prácticas profesionales y su calendarización volvió a ser semestral.

La última modificación al Plan de Estudios de la Carrera de Biología se llevó a cabo en agosto de 1996 (Universidad de Guadalajara, 1996), para instaurar el sistema de créditos, el programa está constituido por cuatro áreas de formación académica: Área Básica Común, en la que se consideran materias básicas también para la licenciatura de Ingeniero Agrónomo y para Medicina Veterinaria y Zootecnia; Área Básica Particular, materias que no se comparten con otras licenciaturas pero básicas para el ejercicio de la profesión; Especializantes, destinadas a profundizar el conocimiento de un área en particular y Optativas Abiertas, materias totalmente selectivas orientadas a incrementar el conocimiento en un área específica de la profesión. El sistema de estudio es semiflexible por lo que el alumno puede terminar su carrera de cuatro a seis años. En este plan no existen orientaciones profesionales definidas, sino que, dependiendo del interés del estudiante, éstas se estructuran en forma conjunta con los tutores, coordinador de carrera y el alumno.

El 20 de julio de 2006 el Consejo General Universitario del Centro Universitario de la Costa firmó el dictamen de creación de la Licenciatura en Biología en ese campus con base tanto en las demandas institucionales como en aquellas contempladas dentro del Plan Nacional de Desarrollo 2001-2006, y en congruencia con las necesidades locales y regionales. La primera generación ingresa en el ciclo escolar 2007A.

Tomando como base el avance científico y tecnológico, en conjunto con la globalización, han obligado a las instituciones de educación superior a realizar reformas para orientar los sistemas de enseñanza hacia la formación en competencias profesionales. La Universidad de Guadalajara a partir de 1999, inicia el proceso de sensibilización e instrucción de sus profesores en torno al rediseño curricular por competencias profesionales, con la motivación principal de elevar la calidad y posibilidades de desarrollo de sus egresados.

Como consecuencia de las necesidades laborales actuales, desde julio de 2010, se comenzó con el trabajo para el rediseño curricular por competencias en el que ambos Centros Universitarios han participado, siendo el reto, además de la distancia, desarrollar actividades de manera conjunta considerando las necesidades de cada sede, en particular la generación de la malla curricular, la definición de las unidades de aprendizaje, procesos nodales, temas nodales y desarrollo de las cartas descriptivas, en respuesta a las características propias de cada CU. 


\section{Malla Curricular y Perfil de Egreso}

Para el trabajo del Análisis y reestructuración Curricular de la Licenciatura en Biología se crearon tres comités;

- Comité de Análisis y Reestructuración Curricular Intercentros formado por 4 integrantes del CUCBA y 4 del CUCosta.

- Comité de Análisis y Reestructuración Curricular del CUCBA.

- Comité de Análisis y Reestructuración Curricular del CUCosta.

Para hacer la propuesta de malla curricular y el perfil de egreso, se realizaron análisis sobre las problemáticas que resuelve el profesional de la biología, los avances científicos y tecnológicos de las ciencias biológicas, líneas y ámbitos de intervención, así como, las recomendaciones del Comité para la Acreditación y Certificación de la Licenciatura en Biología (CACEB) y lo establecido en el Examen General de Certificación Profesional de CENEVAL y se tomó en cuenta la opinión de egresados, comité Consultivo, empleadores, profesores y alumnos con respecto a las competencias que consideraban necesarios en los egresados.

\section{Problemática global en torno a las ciencias biológicas}

Como resultado e integración de estos análisis se definió como problemática global el deterioro de la estructura y función de los sistemas biológicos, desde el nivel molecular hasta el de ecosistemas, así como de los servicios ambientales que proveen. Lo anterior como consecuencia del desconocimiento y la sobreexplotación de los sistemas biológicos, así como por la gestión ambiental errática, que redundan en la pérdida de la biodiversidad, el cambio climático y un uso no sustentable de los recursos naturales, condiciones todas, que afectan la calidad de vida.

\section{Líneas de Intervención y competencias genéricas}

Generación de conocimiento (investigación), Sistematización del conocimiento tradicional o empírico (investigación), Transferencia del conocimiento (educación), Divulgación del conocimiento (divulgación), Elaboración de diagnósticos (investigación y gestión), Diseño e implementación de estrategias resolutivas (gestión e innovación).

\section{Perfil de egreso}

El biólogo egresado de la Universidad de Guadalajara se caracteriza por ser un profesional honesto, tenaz, con una visión analítica y con conciencia crítica, con capacidad de trabajo en equipo, comprometido con el equilibrio naturalezasociedad, que busca promover la calidad de vida de los seres vivos, incluyendo las comunidades humanas, a través de diversas líneas de acción desde el ámbito local hasta el global.

1. Colabora en proyectos de investigación científica acerca de la estructura y función de los sistemas biológicos para comprender la evolución, su funcionamiento y procesos patológicos, y su respuesta a los cambios del ambiente.
2. Elabora diagnósticos sobre la diversidad biológica para conocer el estado de conservación y los servicios ecosistémicos que brinda.

3. Colabora en el diseño de estrategias de conservación y manejo de la biodiversidad y los recursos naturales, teniendo como base el contexto histórico-cultural de la región.

4. Diseña medidas para la prevención, regulación y mitigación del impacto ambiental derivado de las actividades humanas.

5. Aplica e innova procesos biotecnológicos que coadyuven a la conservación y manejo de los servicios ecosistémicos.

6. Desarrolla estrategias de educación, difusión y divulgación científica, para comunicar, educar y sensibilizar a la sociedad acerca de los sistemas biológicos, su evolución, su funcionamiento y procesos patológicos, y su respuesta a los cambios del ambiente.

Una vez establecido el perfil de egreso se definió la plataforma para la construcción de la malla curricular considerando tres áreas (Cuadro 1).

Cuadro 1. Áreas consideradas para la malla curricular

\begin{tabular}{|c|c|c|}
\hline Área básica & Área disciplinar & Área selectiva \\
\hline $\begin{array}{l}\text { 1. Desarrollo de habilidades } \\
\text { de comunicación. } \\
2 \text { Adquisición de } \\
\text { conocimiento de ciencias } \\
\text { básicas auxiliares. } \\
\text { 3.Adquisición del } \\
\text { conocimiento básico de los } \\
\text { sistemas biológicos. } \\
\text { 4. Dominio de una lengua } \\
\text { extranjera. } \\
\text { 5. Desarrollo del } \\
\text { pensamiento científico. } \\
\text { 6. Reconocimiento de los } \\
\text { problemas Conocimiento de } \\
\text { la sociedad y el estado }\end{array}$ & $\begin{array}{l}\text { 1. Adquisición de } \\
\text { conocimiento acerca de la } \\
\text { estructura, función, } \\
\text { evolución y filogenia de los } \\
\text { sistemas biológicos y su } \\
\text { respuesta a los cambios del } \\
\text { ambiente a diferentes } \\
\text { escalas (desde lo molecular } \\
\text { hasta los ecosistemas). } \\
2 \text { Desarrollo de } \\
\text { habilidades para la } \\
\text { investigación, } \\
\text { innovación, la gestión, la } \\
\text { divulgación científica y la } \\
\text { educación }\end{array}$ & $\begin{array}{l}\text { 1. Desarrollo de } \\
\text { Investigación científica, a } \\
\text { través de modelos } \\
\text { biológicos, para la } \\
\text { resolución de problemas que } \\
\text { amenazan la integridad de } \\
\text { servicios ecosistémicos. } \\
\text { 2. Elaboración de } \\
\text { diagnósticos acerca del } \\
\text { estado de conservación de la } \\
\text { diversidad biológica y } \\
\text { estrategias de manejo de los } \\
\text { recursos naturales. } \\
\text { 3. Diseño de estrategias para } \\
\text { la conservación y manejo de } \\
\text { la diversidad biológica y los } \\
\text { servicios ecosistémicos que } \\
\text { proveen en un contexto } \\
\text { histórico-cultural. } \\
\text { 4. Diseño de medidas para } \\
\text { la prevención y regulación } \\
\text { del impacto ambiental. } \\
\text { 5. Innovación de procesos } \\
\text { biotecnológicos. } \\
\text { 6. Desarrollo de estrategias } \\
\text { de divulgación científica. }\end{array}$ \\
\hline
\end{tabular}

Para el área básica y disciplinar, se definieron 5 ejes de formación: a) ciencias básicas, b) contextual metodológico, c) estructura y función, d) biodiversidad y e) ecología y evolución (Cuadro.1). Esta propuesta de áreas y ejes de formación de la malla curricular, permitió el análisis de manera horizontal y vertical de las unidades de aprendizaje, éstas se delimitaron por eje de formación, para cada eje se establecieron los procesos y temas nodales, que permitirán la construcción del conocimiento y el desarrollo de competencias de manera claramente secuencial, de tal manera que esto permite establecer los prerrequisitos entre unidades de aprendizaje, el tiempo en horas/semana/semestre de cada una de ellas y que los procesos 
Cuadro 2. Ejemplo de propuesta de unidad de aprendizaje con sus procesos y temas nodales a partir de la cual se desarrollaron las cartas descriptivas.

\section{INTRODUCCIÓN AL TRABAJO DE LABORATORIO Y CAMPO}

\begin{tabular}{|c|c|}
\hline Eje de Formación Contextual Metodo & Semestre $1(4 / \mathrm{Hrs} / \mathrm{S} / \mathrm{S})$ \\
\hline Procesos Nodales & Temas Nodales \\
\hline $\begin{array}{l}\text { 1. Utiliza los principales } \\
\text { instrumentos de laboratorio y de } \\
\text { campo, de acuerdo al propósito del } \\
\text { estudio y sistema biológico, para la } \\
\text { descripción de la estructura y las } \\
\text { variables de interés. }\end{array}$ & $\begin{array}{l}\text { Materiales, instrumentos y equipos del laboratorio y de campo. } \\
\text { Manejo adecuado de los materiales de cristalería. } \\
\text { Características y fundamento de los equipos del laboratorio } \\
\text { (microscopio, estereoscopio, potenciómetro, balanza, centrifuga, } \\
\text { autoclave, micrótomo, etc.). } \\
\text { Características y fundamento de los equipos de campo (redes, } \\
\text { cuadrantes, botellas, dragas, estaciones meteorológicas, } \\
\text { potenciómetro de campo, refractómetro, sensores de luz, etc.). } \\
\text { Fotografia. } \\
\text { Conceptos básicos de fotografia en laboratorio. } \\
\text { Conceptos básicos de fotografia en campo. }\end{array}$ \\
\hline $\begin{array}{l}\text { 2. Registra la información obtenida } \\
\text { en laboratorio y campo, utilizando } \\
\text { técnicas para su integración e } \\
\text { interpretación de los procesos } \\
\text { biológicos. }\end{array}$ & $\begin{array}{l}\text { Anotaciones científicas. Bitácoras. Notas de campo. Cuadernos. } \\
\text { Bocetos básicos. Hoja de registro. Trabajo de campo. Preparación de } \\
\text { salidas de campo: Elementos a considerar. }\end{array}$ \\
\hline $\begin{array}{l}\text { 3. Reconoce medidas de } \\
\text { bioseguridad, bioética y legislación } \\
\text { vigentes para el manejo responsable } \\
\text { de muestras biológicas que garantice } \\
\text { resultados confiables, sin riesgos } \\
\text { ambientales y personales. }\end{array}$ & $\begin{array}{l}\text { Medidas de bioseguridad en el laboratorio y campo para prevenir } \\
\text { accidentes (manejo de muestras biológicas, reactivos químicos, } \\
\text { desecho de residuos peligrosos y zoonosis). Protección para trabajar } \\
\text { en el laboratorio y campo. Códigos y hojas de seguridad para el } \\
\text { manejo de reactivos químicos. Identificar el manejo apropiado de } \\
\text { Residuos Peligrosos y Biológico Infecciosos (RPBI). Normatividad } \\
\text { vigente para trabajar con seguridad en el laboratorio y campo. }\end{array}$ \\
\hline $\begin{array}{l}\text { 4. Identifica los métodos adecuados } \\
\text { de acuerdo al objetivo del estudio } \\
\text { que aseguren resultados confiables } \\
\text { en estudios de laboratorio y campo. }\end{array}$ & $\begin{array}{l}\text { Métodos adecuados de muestreo y análisis para asegurar resultados } \\
\text { confiables. } \\
\text { Muestras biológicas. } \\
\text { Análisis de muestras biológicas. } \\
\text { Recolección de muestras en laboratorio y campo. } \\
\text { Características necesarias para obtener muestras de calidad. } \\
\text { Seleccionar el método de análisis apropiado para las diferentes } \\
\text { muestras biológicas (agua, fluidos biológicos, muestras vegetales, } \\
\text { suelo, aire, etc.). }\end{array}$ \\
\hline
\end{tabular}

o temas nodales no se repitieran y fueran los necesarios para cumplir con el perfil de egreso (Cuadro 2).

Por otro lado, el desarrollo de estos documentos base, tanto la malla curricular como los procesos y temas nodales, permitirían un óptimo desarrollo de las cartas descriptivas de cada unidad de aprendizaje para que las características establecidas en el perfil de egreso se logren de manera adecuada.

El análisis de la propuesta de Malla Curricular de la Licenciatura en Biología se llevó a cabo de manera colegiada, con la participación de todo el profesorado, este análisis fue realizado por eje de formación, y contó con la participación de profesores de las diferentes academias establecidas en los Centros Universitarios, una vez que fueron analizados los ejes, reestructurados y o aceptados con sus procesos y temas nodales, la elaboración de las cartas descriptivas estuvo a cargo de las academias correspondientes. Los comités de análisis y reestructuración curricular fueron los encargados de revisar y valorar que los procesos y temas nodales hayan sido considerados en las cartas descriptivas.

En ambos Centros Universitarios se impartió el Curso de Diseño de Unidades de Aprendizaje y como producto de estos cursos se desarrolló una propuesta consensuada de formato para la Carta Descriptiva de las Unidades de Aprendizaje, entre ambos centros y aprobada por el Comité de Análisis y Reestructuración Curricular Intercentros.

Se establecieron como acuerdos los siguientes:

a) Establecer prerrequisitos de las unidades de aprendizaje que lo requieran.

b) Una vez que los estudiantes seleccionen un área terminal deberán cumplir con las unidades de aprendizaje la constituyen.

c) Los estudiantes podrán elegir un área terminal independientemente de su Centro Universitario de origen, es decir, se pretende que desde el dictamen quede establecida la movilidad intercentros.

d) Cada Centro Universitario establecerá sus áreas terminales y un rasgo del perfil del egresado por área terminal.

\section{Comentarios finales}

Lo aquí expuesto representa sólo una breve descripción del trabajo que implicó el llevar a cabo el proceso de reestructura- 
ración curricular del Programa Educativo de Licenciatura en Biología de la Universidad de Guadalajara.

$\mathrm{Si}$ bien es cierto que no ofrece una ruta pormenorizada de los pasos a llevar a cabo para un trabajo colegiado de esta naturaleza, sí permite identificar los elementos centrales a tomar en cuenta al momento de emprender un proyecto de actualización curricular.

En lo que respecta a los retos que como comité curricular intercentros debimos enfrentar, es posible identificarlos desde dos áreas específicas; siendo la primera el reto de la distancia geográfica, que si bien fue solventada al contar con un respaldo institucional que permitió poder desarrollar un gran número de reuniones en las cuales se pudo trabajar de manera presencial y cercana entre todos los integrantes del Comité Intercentros, esto implicó un esfuerzo mayúsculo relacionado con el traslado que debían llevar a cabo los integrantes del CUCosta, a la vez que no propiciaba tener una interacción con el resto de los profesores participantes en el proceso, tanto del CUCBA como del CUCosta. Por otro lado, la diferencia existente entre ambos centros universitarios, respecto a los años que llevan ofreciendo la Licenciatura, plantea asimetrías tanto en la infraestructura como en la planta docente, como en el número de estudiantes totales y de primer ingreso.

Lo que conlleva a que la implementación de la propuesta curricular presente variaciones que responderán a las características propias de cada centro universitario.

Al momento en que se presenta este documento la modificación curricular de la Licenciatura en Biología ya ha sido implementada en ambos centros universitarios. Se ha dado continuidad al trabajo colegiado entre ambos centros universitarios, ahora a través de un Comité de Implementación y Seguimiento Intercentros, cuyo principal objetivo es llevar a cabo procesos de evaluación y análisis que incorporen los procesos propios de evaluación, en las respectivas academias, como por ejemplo en lo que respecta a la implementación de las cartas descriptivas de cada una de las Unidades de Aprendizaje que conforman el plan de estudios (https://drive.google.com/drive/folders/1ln5Y3GgHV0X9c7d

TOvliNzFvwC-jsklx?usp=sharing). Cabe mencionar que el plan de estudios vigente se aprobó en consejo general Universitario el 15 de diciembre de 2017 con el dictamen número. I/2017/273, para operar a partir del ciclo 2018B. Como cada programa educativo siempre se puede mejorar, es por ello que a partir del ciclo 2021B, estaremos analizando las cartas descriptivas de estos primeros semestres para su evaluación y mejora. Así mismo, debido a las condiciones actuales de trabajo de manera virtual, se está trabajando para operar con una modalidad mixta. Sin duda, un trabajo arduo donde han participado las academias y comités correspondientes de ambos centros universitarios.

\section{Literatura citada.}

- CONANP. s.a. Sierra de Manantlán a la Vanguardia. http://manantlan.conanp.gob.mx/

- Mendoza C. Historia de la Universidad de Guadalajara.

- Universidad de Guadalajara. 1996.

- Universidad de Guadalajara. 2000 Dictamen de Modificación de Plan de Estudios de la Licenciatura en Biología. http://www.hcgu.udg.mx/dictamenes/dictamenno-i2000739

- Universidad de Guadalajara. 2004. Presentación. Departamento de Estudios para el Desarrollo Sustentable de Zonas Costeras. http://www.costera.melaque.udg.mx/ presenta.html

- Universidad de Guadalajara. 2010. Historia. Centro Universitario de la Costa. http://www.cuc.udg.mx/historia

- Universidad de Guadalajara. 2021. Presentación. https://www.udg.mx/es/nuestra/presentacion 
Cuadro 3. Malla curricular plan de estudios actual en vigencia a partir del calendario 2018B

\begin{tabular}{|c|c|c|c|c|c|c|c|c|}
\hline \multicolumn{9}{|c|}{ SEMESTRES } \\
\hline I & II & III & IV & $\mathbf{V}$ & VI & VII & VIII & $\mathbf{L X}$ \\
\hline $\begin{array}{l}\text { Quimica } \\
8 \mathrm{hrs} / 12 \mathrm{C}\end{array}$ & $\begin{array}{c}\text { Bioquimic } \\
\text { a } \\
6 \mathrm{hrs} / 10 \\
\mathrm{C}\end{array}$ & $\begin{array}{c}\text { Biologia } \\
\text { celular } \\
6 \mathrm{hrs} / 10 \mathrm{C}\end{array}$ & $\begin{array}{c}\text { Biologia } \\
\text { molecular } \\
6 \mathrm{hrs} \\
/ 10 \mathrm{C}\end{array}$ & $\begin{array}{c}\text { Genética } \\
\text { I } \\
4 \mathrm{hrs} / \\
7 \mathrm{C}\end{array}$ & $\begin{array}{c}\text { Genética II } \\
4 \mathrm{hrs} / 7 \mathrm{C}\end{array}$ & $\begin{array}{l}\text { Seminari } \\
\text { o de } \\
\text { investigac } \\
\text { ión } \\
\text { biológica } \\
4 \text { hrs / } 6 \mathrm{C}\end{array}$ & $\begin{array}{l}\text { Seminario } \\
\text { de } \\
\text { proyectos } \\
\text { de } \\
\text { intervenció } \\
\text { n en c5. } \\
\text { biológicas } \\
4 \text { hrs } 6 \mathrm{C}\end{array}$ & \multirow{12}{*}{$\begin{array}{c}\text { Práctica } \\
\text { profesio } \\
\text { nal }\end{array}$} \\
\hline $\begin{array}{c}\text { Fisica } \\
\text { aplicada a } \\
\text { las ciencias } \\
\text { biológicas } \\
6 \text { hrs } / 10 \mathrm{C}\end{array}$ & $\begin{array}{l}\text { Fisicoqui } \\
\text { mica } \\
4 \mathrm{hrs} / 7 \mathrm{C}\end{array}$ & $\begin{array}{l}\text { Comunica } \\
\text { ción de las } \\
\text { ciencias } \\
\text { biológicas } \\
4 \mathrm{hrs} / 7 \mathrm{C}\end{array}$ & $\begin{array}{c}\text { Histologi } \\
\text { a y } \\
\text { anatomia } \\
\text { vegetal } \\
6 \mathrm{hrs} \\
/ 10 \mathrm{C} \\
\end{array}$ & $\begin{array}{c}\text { Desarrol } \\
\text { lo } \\
\text { vegetal } \\
4 \mathrm{hrs} / \\
7 \mathrm{C}\end{array}$ & $\begin{array}{c}\text { Fisiologia } \\
\text { vegetal } \\
6 \mathrm{hrg} / 10 \mathrm{C}\end{array}$ & $\begin{array}{c}\text { Evolució } \\
\mathrm{n} \\
4 \mathrm{hrs} / 7 \mathrm{C}\end{array}$ & $\begin{array}{c}\text { Desarrollo } \\
\text { de } \\
\text { emprended } \\
\text { ores } \\
4 \mathrm{hrs} / 6 \mathrm{C}\end{array}$ & \\
\hline $\begin{array}{l}\text { Ciencias de } \\
\text { la Tierra I } \\
4 \mathrm{hrg} / 7 \mathrm{C}\end{array}$ & $\begin{array}{c}\text { Ciencias } \\
\text { de la } \\
\text { Tierra II } \\
4 \mathrm{hrs} / 7 \mathrm{C}\end{array}$ & $\begin{array}{l}\text { Sociedad- } \\
\text { naturaleza } \\
4 \mathrm{hrs} / 7 \mathrm{C}\end{array}$ & $\begin{array}{c}\text { Histologi } \\
\text { a y } \\
\text { embriolo } \\
\text { gia } \\
\text { animal } \\
6 \mathrm{hrs} \\
/ 10 \mathrm{C} \\
\end{array}$ & $\begin{array}{c}\text { Anatomi } \\
\text { a y } \\
\text { fisiologi } \\
\text { a animal } \\
\text { compara } \\
\text { da } \\
8 \mathrm{hrs} / \\
12 \mathrm{C} \\
\end{array}$ & $\begin{array}{c}\text { Introducci } \\
\text { ón a la } \\
\text { biotecnolo } \\
\text { gia } \\
4 \mathrm{hrs} / 7 \mathrm{C}\end{array}$ & $\begin{array}{c}\text { Paleobiol } \\
\text { ogia } \\
4 \mathrm{hrs} / 7 \mathrm{C}\end{array}$ & $\begin{array}{l}\text { Optativa l } \\
4 \mathrm{hrs} / 6 \mathrm{C}\end{array}$ & \\
\hline $\begin{array}{l}\text { Biomatemá } \\
\text { ticas } \\
6 \mathrm{hrs} / 10 \mathrm{C}\end{array}$ & $\begin{array}{c}\text { Bioestadis } \\
\text { tica } \\
6 \mathrm{hrs} / 9 \mathrm{C}\end{array}$ & $\begin{array}{c}\text { Diseños } \\
\text { experiment } \\
\text { ales } \\
6 \mathrm{hrs} / 10 \mathrm{C}\end{array}$ & $\begin{array}{c}\text { Educació } \\
n \\
\text { ambiental } \\
4 \mathrm{hrs} / 7 \mathrm{C}\end{array}$ & $\begin{array}{c}\text { Ecologia } \\
\text { de } \\
\text { poblacio } \\
\text { nes } \\
6 \mathrm{hrs} \\
/ 10 \mathrm{C}\end{array}$ & $\begin{array}{l}\text { Ecologia } \\
\text { de } \\
\text { comunidad } \\
\text { es y } \\
\text { ecosistema } \\
5 \\
6 \mathrm{hrs} / 10 \mathrm{C}\end{array}$ & $\begin{array}{c}\text { Biogeogr } \\
\text { afia } \\
4 \mathrm{hrs} / 7 \mathrm{C}\end{array}$ & $\begin{array}{l}\text { Optativa } 2 \\
4 \mathrm{hrs} / 6 \mathrm{C}\end{array}$ & \\
\hline $\begin{array}{c}\text { Introducció } \\
\mathrm{n} \text { al trabajo } \\
\text { de } \\
\text { laboratorio } \\
\text { y campo } \\
4 \mathrm{hrs} / 7 \mathrm{C}\end{array}$ & $\begin{array}{c}\text { Metodolo } \\
\text { gía de la } \\
\text { investigac } \\
\text { ión en } \\
\text { Ciencias } \\
\text { Biológica } \\
\mathrm{s} \\
4 \mathrm{hrs} / 7 \mathrm{C} \\
\end{array}$ & $\begin{array}{c}\text { Microbiolo } \\
\text { gia } \\
4 \mathrm{hrs} / 7 \mathrm{C}\end{array}$ & $\begin{array}{c}\text { Hongos } \\
6 \mathrm{hrs} \\
/ 10 \mathrm{C}\end{array}$ & $\begin{array}{c}\text { Algas, } \\
\text { briofitas } \\
y \\
\text { pteridofi } \\
\text { tas } \\
6 \mathrm{hrs} \\
/ 10 \mathrm{C} \\
\end{array}$ & $\begin{array}{c}\text { Gimnosper } \\
\text { mas y } \\
\text { angiosper } \\
\text { mas } \\
6 \mathrm{hrs} / 10 \mathrm{C}\end{array}$ & $\begin{array}{c}\text { Obligator } \\
\text { ia l } \\
4 \mathrm{hrs} / 6 \mathrm{C}\end{array}$ & $\begin{array}{c}\text { Selectiva l } \\
4 \mathrm{hrs} / 6 \mathrm{C}\end{array}$ & \\
\hline $\begin{array}{c}\text { Biologia } \\
\text { general } \\
6 \mathrm{hrs} / 10 \mathrm{C}\end{array}$ & $\begin{array}{c}\text { Taxonomi } \\
\mathrm{a} \\
6 \mathrm{hrs} / 10 \mathrm{C}\end{array}$ & $\begin{array}{c}\text { Protozoari } \\
\text { os } \\
4 \mathrm{hrs} / 7 \mathrm{C}\end{array}$ & $\begin{array}{c}\text { Invertebr } \\
\text { ados no } \\
\text { artrópodo } \\
\mathrm{g} \\
6 \mathrm{hrs} / \\
10 \mathrm{C}\end{array}$ & $\begin{array}{c}\text { Artrópo } \\
\text { dos } \\
6 \mathrm{hrs} \\
/ 10 \mathrm{C}\end{array}$ & $\begin{array}{l}\text { Cordados } \\
6 \mathrm{hrs} / 10 \mathrm{C}\end{array}$ & $\begin{array}{l}\text { Obligator } \\
\text { ia } 2 \\
4 \mathrm{hrs} / 6 \mathrm{C}\end{array}$ & $\begin{array}{l}\text { Selectiva l } \\
4 \mathrm{hrs} / 6 \mathrm{C}\end{array}$ & \\
\hline \multicolumn{6}{|c|}{ Eje de formación de ciencias básicas } & \multirow{3}{*}{$\begin{array}{l}\text { Obligator } \\
\text { ia } 3 \\
4 \mathrm{hrs} / 6 \mathrm{C}\end{array}$} & \multirow{3}{*}{$\begin{array}{l}\text { Tópico } \\
\text { selecto } 1 \\
4 \text { hrs } 6 \mathrm{C}\end{array}$} & \\
\hline & Eje de & formación es & rructura-func & & & & & \\
\hline \multicolumn{6}{|c|}{ Eje de formación contextual metodológico } & & & \\
\hline \multicolumn{6}{|c|}{ Eje de formación biodiversidad } & \multirow{3}{*}{$\begin{array}{c}\text { Obligator } \\
\text { ia } 4 \\
4 \mathrm{hrs} / 6 \mathrm{C}\end{array}$} & \multirow{3}{*}{$\begin{array}{l}\text { Tópico } \\
\text { selecto } 1 \\
4 \mathrm{hrs} / 6 \mathrm{C}\end{array}$} & \\
\hline & Eje de $f$ & ormación eco & ogia y evol & & & & & \\
\hline \multicolumn{6}{|c|}{ Orientación } & & & \\
\hline \multicolumn{9}{|c|}{ CREDITOS } \\
\hline 56 & 50 & 48 & 57 & 56 & 54 & 51 & 48 & 30 \\
\hline
\end{tabular}

